\title{
Criminologie
}

\section{Gangs juvéniles et construits ethniques dans le contexte américain}

\section{Laurence Tichit}

Volume 36, numéro 2, automne 2003

Le construit de l'ethnicité en criminologie

URI : https://id.erudit.org/iderudit/007866ar

DOI : https://doi.org/10.7202/007866ar

Aller au sommaire du numéro

\section{Éditeur(s)}

Les Presses de l'Université de Montréal

ISSN

0316-0041 (imprimé)

1492-1367 (numérique)

Découvrir la revue

Citer cet article

Tichit, L. (2003). Gangs juvéniles et construits ethniques dans le contexte américain. Criminologie, 36(2), 57-68. https://doi.org/10.7202/007866ar
Résumé de l'article

En nous appuyant sur une revue de la littérature criminologique essentiellement américaine à propos des gangs de rue, nous interrogerons la construction ethnicisante, voire racialiste des conduites délinquantes collectives des jeunes issus des minorités, en prenant l'exemple des gangs afro-américains. Ceci en ramenant cette construction à deux lignes d'analyse dont la première est celle du contexte historico-national de production de l'objet " gangs de rue " et de l'ethnicité qui y est associée, avec les notions de " relations raciales » et d'« interethnicité ". La seconde est celle de l'agir ethnique, s'observant sur les deux faces interne et externe de la communalisation. C'est la construction ethnique de l'individu dans le rapport qu'il entretient avec sa communauté idéelle d'appartenance, sa culture et son histoire, et simultanément, le rapport qu'il entretient avec les autres, dans un double mouvement de désignation et d'autodésignation, traçant une frontière labile, parfois statique, entre les « eux » et les " nous ».
Ce document est protégé par la loi sur le droit d'auteur. L'utilisation des services d’Érudit (y compris la reproduction) est assujettie à sa politique d'utilisation que vous pouvez consulter en ligne.

https://apropos.erudit.org/fr/usagers/politique-dutilisation/ 


\title{
Gangs juvéniles et construits ethniques dans le contexte américain
}

\author{
Laurence Tichit \\ Stagiaire postdoctorale \\ Centre international de criminologie comparée \\ Université de Montréal \\ tichit@aol.com
}

RÉSUMÉ • En nous appuyant sur une revue de la littérature criminologique essentiellement américaine à propos des gangs de rue, nous interrogerons la construction ethnicisante, voire racialiste des conduites délinquantes collectives des jeunes issus des minorités, en prenant l'exemple des gangs afro-américains. Ceci en ramenant cette construction à deux lignes d'analyse dont la première est celle du contexte historico-national de production de l'objet «gangs de rue » et de l'ethnicité qui y est associée, avec les notions de «relations raciales» et d'«interethnicité». La seconde est celle de l'agir ethnique, s'observant sur les deux faces interne et externe de la communalisation. C'est la construction ethnique de l'individu dans le rapport qu'il entretient avec sa communauté idéelle d'appartenance, sa culture et son histoire, et simultanément, le rapport qu'il entretient avec les autres, dans un double mouvement de désignation et d'autodésignation, traçant une frontière labile, parfois statique, entre les «eux» et les «nous».

ABSTRACT - Considering an array of mainly North-American scientific works on street gangs, we will examine the ethnicized and racialized construction of minority-issued youth groups' offending behaviour, focusing on African-American gangs. We will consider this issue from two points of view. The first one will be the national and historic creation of the «street gang » object/subject and its related ethnicity, combined with notions of racial relationships and inter-ethnicity. The second perspective will study ethnic behaviour in terms of inner and outer communalization. We will study one's ethnic construction through one's relationship with an ideal community, one's culture and history, and simultaneously, the relationship one has with others, in a twofold movement of identification and auto-identification, drawing an indefinite line, sometimes an unwavering one, between «Them» and «Us».

Régulièrement, des actes de délinquance et de violence fortement médiatisés et imputés à des jeunes constitués en gangs de rue, reviennent sur le devant de la scène publique de la criminalité juvénile. Et d'une façon 
tout aussi récurrente, la dimension ethnique desdits groupes est interrogée, qu'elle le soit à travers la rhétorique multiculturaliste ou qu'elle serve de point d'appui au vent de la «tolérance zéro» ou au discours sécuritaire, parfois électoralement porteur, de l'intégration assimilationniste républicaine. D'une vision dynamique de l'ethnicité, nous entrons peu à peu dans une ethnicisation, voire une racialisation des rapports sociaux, par l'importance accordée à la caractérisation ethnico-raciale. L'ethnicisation des relations sociales n'est cependant qu'une face de l'ethnicité, à laquelle elle ne se réduit pas. Alors que l'ethnicité ouvre un jeu de cartes identitaires possible, l'ethnicisation, elle, fait «système». L'objet de cet article est alors d'interroger cette ethnicisation représentée ici par la figure des gangs de rue, dans les fonctions sociales et politiques qui leur sont attribuées et qu'ils s'attribuent. Ceci en replaçant les débats théoriques autour de l'objet «gangs de rue» dans les contextes historico-nationaux et les rapports sociopolitiques dont le rapport à l'État et à la société civile dans lesquels ils prennent sens, à partir du moment où la délinquance juvénile collective est définie à différents degrés de priorités, en termes de «problème de sécurité publique».

\section{Fondements d'une école sociologique : les gangs à Chicago}

Le premier intérêt scientifique pour les gangs de rue est celui né dans la tradition américaine de l'École de Chicago des deux phases des années 1930 et 1950-1960. Il s'inscrit à l'origine dans une vague criminelle et un contexte récurrent de conflits entre gangs à Chicago, objet d'une vaste enquête sur la criminalité, en 1924, lancée par l'Illinois Association for Criminal Justice (Landesco, 1929). À partir de cette source, plusieurs lignes théoriques interprétatives ont été proposées. En premier lieu, celle qui s'appuie sur la théorie de la «désorganisation sociale» pour expliquer l'apparition de gangs dans des zones interstitielles de la société en milieu urbain (Trasher, 1927; Shaw et McKay, 1942). Selon Trasher (1927), les gangs sont l'expression de l'affaiblissement du contrôle social, révélant ainsi une crise sociale profonde dans la fraction la plus démunie de la population américaine, dont les groupes d'immigrants nouvellement installés. Les gangs sont ici appréhendés comme une forme temporaire et transitoire d'organisation de jeunes à terme intégrés. Le concept central ici de «désorganisation sociale» sera remis en cause notamment par Whyte (1943), avec son étude en observation participante d'un quartier 
italien pauvre de Boston, où il montre qu'il y règne une organisation complexe basée sur un système d'obligations réciproques. Selon l'auteur, ce quartier est en réalité organisé, mais d'une façon différente. À partir des années 1950, apparaissent des théories prenant appui sur les théories «sous-culturelles» et sur les théories de la «tension» (Merton, 1938; Parsons, 1954). Ici, l'objet de recherche est la relation entre une sousculture délinquante juvénile transmise par les pairs et la source de la délinquance définie par un problème d'ajustement et de tension entre structures sociales et valeurs culturelles dominantes de la société. Il est question ici de statut (Fillieule, 2001). Ce sont les travaux de Cohen (1955), puis de Cloward et Ohlin (1960), qui étudient le processus d'apparition de différents types de sous-cultures délinquantes, en fonction des contextes dans lesquels se trouvent placés les membres des gangs. Les auteurs tentent ainsi de rendre compte de trois types de sous-culture délinquante et orientations des gangs, sans qu'elles soient exclusives : les gangs criminels, les gangs conflictuels et les gangs retraitistes. Ces présupposés culturalistes seront questionnés entre autres par Matza (1964), quant à un certain «déterminisme» de la délinquance comme valeur que les notions de sous-culture sous-tendent et leur nature que leur force oppositionnelle aux règles légales (Fillieule, 2001). Enfin, un autre champ issu de l'École de Chicago, est la théorie interactionniste de l'étiquetage ou du «labelling», qui permet de comprendre comment la «désignation» d'un individu en tant que déviant, peut l'amener à se regrouper avec d'autres individus objets de la même désignation, forgeant ainsi une identité collective pouvant prendre parfois la forme d'un gang et en adopter les logiques (Becker, 1963).

Les perceptions des gangs de rue à travers ces premiers travaux et analyses, mettent ainsi l'accent sur des notions culturalistes, de statut et de transition vers l'âge adulte. L'une des façons d'envisager le phénomène des gangs de rue, serait donc de le lire à travers le prisme du développement adolescent. Le regroupement grégaire juvénile y est l'une des fonctions socialisatrices essentielles. Dans et par le groupe, l'adolescent apprend et se confronte aux règles oppositionnelles et identitaires appartenant à «l'école de la vie» du monde des pairs. Les gangs juvéniles ne seraient pas ainsi une «nouveauté». Cependant, du groupe, à la bande de quartier ou au gang, il est des distinctions à établir : tout groupement juvénile ne constitue pas un gang, tout comme il peut l'être. De plus, l'évolution des gangs eux-mêmes est liée à la perception sociale du phénomène, par sa visibilité groupale et les degrés et les formes de «panique 
morale» (Moore, 1993) qu'il suscite. La culture de la peur n'existe que dans son rapport à la culture de la violence et vice-versa. Et l'épaisseur historique de la dangerosité sociale ou de la force intégrative imputée à des manifestations collectives juvéniles reste encore, selon nous, largement à analyser dans leurs manifestations contemporaines, si l'on considère que la "panique morale» se construit dans des contextes déterminés autour de clivages préexistants de classes et/ou de races.

\section{La contemporanéité des gangs de rue}

Après l'éclipse des années 1970, la thématique des gangs de rue connait une renaissance aux États-Unis dans les années 1980, avec une reprise de la recherche en partie suscitée par le gouvernement fédéral américain, inquiet de la situation jugée alarmante, notamment à Los Angeles. C'est par la violence, l'augmentation des crimes violents, notamment des homicides, et l'utilisation de plus en plus répandue des armes à feu (Risbil et Davidson 1993; Stephens, 1993; Parks, 1995), l'utilisation d'armes à partir de voitures en marche («drive-by shootings»), la question de la relation des gangs aux trafics de drogue (Skolnick, 1990), puis dans un contexte d'émeutes urbaines (South Central à Los Angeles, en 1992), que se redessine la nouvelle figure des «gangs contemporains», dont les paradigmes théoriques de l'École de Chicago, désormais surannés, auraient du mal à rendre compte. Se recompose également le paradigme urbain remplaçant celui incarné au début du siècle par la ville industrielle de Chicago. Il ressort alors que la figure des gangs contemporains est construite autour de l'observation centrale selon laquelle les gangs ont dans l'ensemble tendance à être peu structurés, donc instables, tout en étant davantage orientés vers la violence, la drogue et les armes. La production scientifique à propos des gangs de rue va prendre dès lors un tournant inflationniste aux États-Unis, soulignant la gravité des problèmes d'ordre public, puis nationaux, que posent les gangs ${ }^{1}$. Monti et Cummings (1993) soulignent d'abord l'effet d'urgence des législateurs et des services de police, rédigeant à la hâte des programmes d'intervention et de lutte contre le phénomène. Progressivement, le «problème gangs de rue» va s'institutionnaliser, par la mise en place en milieu policier d'unités

1. En 1998, une vaste enquête, le National youth gang survey (Moore et Cook, 1998), menée aux États-Unis auprès de 2668 répondants provenant de 660 districts de police, estimait la présence de 28799 gangs juvéniles aux activités délinquantes et de 780000 membres. 
spéciales de repérage, de suivi et d'intervention, et la diffusion de programmes d'information et de formation destinés au public. Cependant, si la proportion d'actes violents commis par des gangs durant les années 1980 et 1990 a pu être importante, la criminalité des gangs demeure faible par rapport à l'ensemble de la criminalité, que ce soit à l'échelle de la ville, des systèmes pénitentiaires ou des écoles (Jankowski, 1994; Spergel, 1995). L'hypothèse d'une modification de la «sensibilité » publique moins tolérante à l'égard de la violence juvénile, combinée à un recours plus fréquent aux services de police qu'auparavant, notamment par les organismes en relation avec des jeunes, et donc une plus grande visibilité et existence de la violence juvénile, peut être évoquée ici. L'hypothèse d'une plus forte utilisation, en réaction à ce phénomène, et d'une plus grande tolérance de la violence par les jeunes, en particulier par les membres de gangs, est à envisager. Cependant, le lien entre violence juvénile et gangs de rue est difficile à établir, étant donné que la catégorisation statistique est d'emblée confrontée au problème de disponibilité de données fiables appelant une standardisation des définitions qui bute sur l'angle d'analyse à privilégier : s'agit-il de prendre en compte les délits et les crimes relevant d'une activités spécifique aux gangs, toute infraction dont l'auteur ou la victime appartiennent à un gang, ou tout acte délictueux commis en groupe? Reste donc, selon nous, à déterminer s'il convient d'étudier la violence juvénile et/ou la violence criminelle des gangs de rue, en leur redonnant l'épaisseur historique, sociologique et pénale dans laquelle elles prennent sens. Notons également la popularisation d'un modèle issu essentiellement du milieu policier, cherchant à dresser une typologie des gangs, considérés comme des organisations socialisatrices et/ou criminogènes et en tout cas normatives. Cela suppose un degré minimum de hiérarchisation, de distribution des rôles, des statuts et des légitimités entre les membres, telles les organisations formelles. La question identitaire passe ici par des attributs d'appartenance, dont le territoire est l'un des éléments forts. Les gangs sont traditionnellement organisés sur des bases territoriales, qu'elles soient de l'ordre d'une appartenance pure et simple, d'occupation et/ou de monopole sur les activités criminelles (Jankowski, 1992). Le centre de décision ou le «noyau dur» forme la «clique» et les leaders ont parfois une appellation formelle («King», «Prince», «Don», «Chief»). Suivent les membres associés et périphériques, puis les membres flottants (les «floaters») ou ayant une spécialisation, et les recrues («wannabes») qui sont des aspirants et des membres potentiels. Enfin, durant les années 1970, des coalitions de 
gangs se sont formées, dessinant les célèbres figures des «nations», telles les «People» (27 gangs) et les «Folks» (31 gangs) de Chicago ou les très médiatiques «Bloods and Crips» de Los Angeles toujours en activité (Jankowski, 1992). Le débat s'organise alors autour de la spécificité de chaque groupe et des tendances actuelles qui peuvent être observées, en termes d'écarts par rapport à cette définition d'un gang fortement structuré et criminalisé. Il est question, entre autres, de modes d'organisation, de leadership, de degré de cohésion des groupes (Klein, 1971) et de l'analyse des contextes dans lesquels se développent les gangs, dont par exemple leurs modalités de recrutement (Johnstone, 1983). Les tendances aux États-Unis depuis les années 1980, outre la question de la violence déjà mentionnée, seraient celles d'une modification des modes opératoires des gangs, telles des opérations peu planifiées à l'avance et commises en petits groupes, l'observation de groupes éphémères et apparemment sans "chef», et le rajeunissement des membres et/ou la présence d'adultes. Enfin, il est question de «l'essaimage» des gangs (Huff, 1996; Hagedorn, 1988), présents dans des villes de taille moyenne, après des décennies d'une implantation limitée aux ghettos situés au cœur des grandes villes telles Los Angeles, Chicago, New York et Philadelphie (Jankowski, 1992).

\section{Un consensus introuvable}

L'obstacle principal auquel sont confrontés les chercheurs serait l'absence d'une définition commune et standardisée des concepts centraux de «gang», de «membre de gang» et d' «incident de gang» (Hébert et al., 1997). Les débats théoriques s'articulent, entres autres dimensions, autour de typologies en fonction de la nature et de la finalité des activités des gangs (gangs à visée idéologique, gangs de territoire, organisations criminelles), de leur degré de criminalité et de leur niveau d'organisation (Spergel, 1995), voire des contextes de vulnérabilité sociétale dans lesquels ils évoluent (Walgrave, 1992) ou des dispositions psychosociales des individus qui y sont attachés (Lanctôt et Leblanc, 1997). S'il en résulte une difficulté à évaluer l'ampleur du phénomène et son évolution dans le temps, ce qui pose des problèmes sur les plans de la prévention, de l'intervention et de la recherche (Parks, 1995; Spergel, 1992; 1995), ceci sous-tend l'idée d'une variabilité des configurations des gangs de rue et de la perméabilité de la vie des groupes que des typologies statiques ne peuvent traduire, hormis pour quelques groupes criminalisés fortement implantés depuis plusieurs années. L'intérêt ici est que le débat autour 
des définitions nous renseigne tout autant sur celui qui classifie que sur les classifications elles-mêmes. Plus précisément, il dévoile les enjeux théoriques, pratiques, idéologiques à l'égard des gangs, des différents groupes d'acteurs construisant les données (chercheurs, corps policiers, administrateurs, groupes communautaires, etc.). Pour exemple, les services de police se dotent d'une définition des gangs de rue mettant l'accent sur les notions de criminalité, d'intimidation et de violence, alors que d'autres acteurs sociaux soulignent la nécessité de considérer les membres des gangs avant tout comme des jeunes, appelant ainsi à une action intégrée. Il s'agit de ramener les discours et les pratiques «savantes» et de «sens commun» construits à propos des gangs, à leur contexte de production et d'analyser comment les forces et les pouvoirs des différents corps professionnels interagissent les uns avec les autres, sur un continuum d'appréhensions des gangs de rue et non sur un consensus introuvable. Ceci suppose le dépassement de l'unique problème des signes extérieurs des activités «criminelles» auquel les gangs de rue ne sont pas réductibles, s'ils sont appréhendés en tant que groupes sociaux, objets et sujets de rapports sociaux et de rapports de domination. Les questions identitaires sont alors une des entrées analytiques parmi d'autres et non la seule, permettant d'en rendre compte. Cependant, en regard des analyses des sociologues de l'École de Chicago des années 1930, puis 1960, il est une évolution importante à souligner. Autant les questions ethniques, voire raciales, à propos des gangs de rue étaient analysées du point de vue d'un espace mouvant de transition ouvert, autant les analyses contemporaines se centrent sur une spatialisation géographique, humaine et sociale tournée vers un présent immuable et statique.

\section{« Black Power », « underclass » ou la condition afro-américaine}

Analyser les gangs de rue par les questions identitaires éclaire la notion d'ethnicisation, voire de racialisation des rapports sociaux, d'une part entre les groupes déviants et le reste de la société et entre les groupes minoritaires et majoritaires, d'autre part. Symbolisant une certaine forme d'appartenance se définissant en creux parmi d'autres appartenances, la question des gangs de rue et de l'ethnicité est ainsi recentrée dans un contexte global, lui donnant sens. Pour ce faire, nous nous limiterons ici aux figures connues et implantées depuis longtemps de gangs de rue rassemblant des adolescents noirs aux États-Unis. Le concept d'ethnicité 
(Poutignat et Streiff-Fenart, 1995 ; Juteau, 1999) rompant avec une vision essentialiste et statique de l'Autre, permet une identification commune plus ou moins définie, pour pouvoir poser une limite provisoire. Il structure des interactions et des rapports sociaux. Dans une perspective dynamique, il désigne tout autant qu'il est désigné, les figures d'acteurs sociaux en conflit autour de plusieurs lignes de tension qui ne sont pas nouvelles mais qui peuvent se radicaliser : l'individuation extrême et de nouvelles formes de socialisation, le jeu du balancier entre l'universel et le particulier. Dans ce jeu de cartes labile, apparaissent des processus de crispation identitaire que nous désignons pour notre part, par «ethnicisation», voire «racialisation» des rapports et des enjeux sociaux (Tichit, 1995; 2001a; 2001b) liés au contexte historico-national dans lequel ils émergent. Avec Martiniello (1995), nous avons une grille d'analyse opératoire à trois niveaux d'appréhension de l'ethnicité. Le niveau individuel de la dimension subjective de l'ethnicité correspondant au sentiment, à la conscience d'appartenance qu'éprouve l'individu à l'égard d'un groupe ${ }^{2}$. Le second niveau groupal et mésosocial, correspond à la mobilisation et à l'action collective. C'est ce que nous appelons pour notre part, «l'agir ethnique». Enfin, le niveau macrosocial englobe les contraintes structurelles de nature sociale, économique et politique qui façonnent les identités ethniques et qui assignent les individus à une position sociale déterminée, en fonction de leur appartenance imputée à une catégorie ethnique. Ici, l'ethnicité n'est plus simplement appréhendée en termes identitaires, l'accent est mis sur les contraintes structurelles qui s'imposent de façon plus ou moins décisive aux individus, entraînant des conséquences sur les différentes dimensions de leur vie quotidienne. En ce sens, l'ethnicité constitue un axe objectif de clivage social dans les sociétés contemporaines (Martiniello, 1995). Or, aux États-Unis, le terme renvoie à une distinction classique de la sociologie, entre minorités immigrées ou minorités ethniques relevant de la problématique de la «nouvelle ethnicité» («ethnicity»)

2. Cette approche s'inspire des travaux de Weber (1971), selon lesquels les groupes ethniques sont des ensembles d'individus qui croient en une communauté d'origine commune fondée sur une similitude de culture, de mœurs ou d'expérience, abstraction faite de l'existence objective d'une telle communauté.

«Mon appartenance à la Bretagne n'est qu'une qualité facultative que je puis parfaitement renier ou méconnaître. Je l'ai d'ailleurs fait. J'ai longtemps ignoré que j'étais breton... je l'ai par moment oublié? Français sans problème, il me faut donc vivre la Bretagne en surplus, ou pour mieux dire, en conscience. Si je perds cette conscience, la Bretagne cesse d'être en moi. Si tous les Bretons la perdent, elle cesse absolument d'être. La Bretagne n'a pas de papiers» (Lebesque, 1970). 
des descendants des immigrés européens, et «minorités raciales», correspondant à la condition des Noirs et des autres minorités de couleur, sujettes aux discriminations et à l'exclusion sociale, relevant des «relations raciales». Selon nous, la problématique des gangs de rue afro-américains trouve son sens dans la position sociohistorique des minorités noires sur cet axe structurel de l'ethnicité. Ils en sont tout à la fois la solution et le problème. Dans un contexte où la moitié des familles vit en dessous des seuils de pauvreté, les activités illégales et les gangs apparaissent comme des systèmes de survie, mais qui engagent les populations pauvres dans une spirale infernale dès lors que le stigmate de la violence renforce les logiques économiques et racistes d'exclusion. Les réseaux de gangs fournissent les plus stables des opportunités économiques offertes aux jeunes issus des minorités dans certaines zones où la seule représentation institutionnelle est parfois celle de la police (Phillips, 1999). De plus, l'option répressive et l'emprisonnement de jeunes leur ont donné paradoxalement l'opportunité de trouver dans la prison une véritable école du crime, structurant les gangs en fédérations dont les célèbres «Bloods and Crips», qui ont disséminé le système de gang dans tout le pays à partir de leur réseau carcéral. Selon Phillips (1999), ce qui caractérise les gangs de jeunes Noirs, en particulier à Los Angeles, est leur plus grande visibilité dans la société américaine. Et ce, selon trois facteurs interdépendants. D'une part, c'est pour les populations noires que l'effet de ghetto, de concentration dans une zone sans emploi et sans services, a été le plus fort. Ce sont également les gangs de jeunes de ces ghettos qui ont été le plus impliqués dans le trafic des drogues dures et des armes à feu, d'autre part. Enfin, c'est entre ces gangs que s'est déchaînée la plus forte violence. Cependant, le gang de rue constitue un lieu et un lien socialisant très attirant pour les jeunes. Il est le reflet de la ségrégation américaine, où les jeunes Noirs des ghettos sont très tôt initiés à la violence et à la lutte pour la survie en milieu urbain (Canada, 1995). Du point de vue de l'agir ethnique, les membres des gangs légitiment leur action en considérant qu'ils appliquent la loi du plus fort d'une société américaine générée par cette même loi et fondée dans et par la violence et les rapports de force entre groupes d'intérêts : «les gangs ont bâti ce pays». S'inspirant $\mathrm{du}$ «Black Power», ils y trouvent la source mythique de leur communalisation et le fondement de leur propre histoire identitaire. La vie de voyou est indissociablement liée à leur condition, elle signifie l'élévation des pauvres et des exclus, tout en exprimant leur propre autodestruction. Les messagers du «gangsta rap» fortement médiatisés, sont devenus l'emblème 
de cette ambivalence. Ils sont l'image poussée à son paroxysme de cette double facette traversant la vie des jeunes Noirs des ghettos américains. Ils sont tout à la fois le mal qu' il faut combattre et le combat contre le mal. D'où tout un arsenal «guerrier» présent dans le mode de vie des gangs. Cela passe par les appellations des groupes, tels des «posse» ${ }^{3}$, des cliques ou des «crew», les règles internes basées sur l'initiation, l'honneur et la loyauté au groupe et les signes extérieurs de différenciation. Cependant, au-delà du récit des origines, les gangs de rue sont le reflet d'une aliénation généralisée, où l'inimitié avec son proche voisin et les alliances et désalliances entre les groupes dans une coupure amis/ennemis, reproduisent en interne les processus de fragmentation et d'exclusion (Phillips, 1999). Les premières victimes sont les jeunes de la communauté ellemême, sujets et objets d'une violence tournée vers soi et d'une guérilla intestine. Finalement, le fait que les gangs de rue utilisent la mythologie $\mathrm{du}$ «Black Power» à des fins strictement délinquantes pour asseoir leur pouvoir de caïdats locaux suit cette même logique d'aliénation, selon nous, en exploitant et détruisant la communauté, en laissant croire en la loi du plus fort, dans un jeu de domination sans fin.

\section{Conclusion}

Oublier les «relations raciales» traversant les gangs de rue de jeunes Noirs américains et les analyser sous l'angle de «tribus», en réactualisant les stéréotypes «ethniques», reviendrait ainsi à les situer dans l'étrangeté et à occulter les rapports de pouvoir, alors qu'ils ne sont pas en marge de la société américaine. Ils posent un miroir ayant la faculté de refléter, donc de voir et de se faire voir. Car la catégorisation ethnicoraciale et les «relations raciales» sont elles aussi, finalement, des construits sociaux et historiques, activés des deux côtés de la délimitation de la frontière. Nous ne faisons pas non plus l'apologie de la recherche à tout prix des traces d'une contre-culture, preuve d'une maturité nouvelle de la jeunesse noire à travers une «communalisation de résistance». Mais nous voulons souligner l'importance de la représentation des forces sociales de ségrégation et d'aliénation, comme signification «d'un non âge de raison sociale» et par conséquent de l'existence d'une irrationalité sociale que l'on a tour à tour, suivant les idéologies dominantes de chaque époque, nommée les «petits pauvres de Dieu» ou les «exploités

3. Au sens littéral, «petite armée». 
du capitalisme» ou les «martyres de la modernité», invariant du non-sens du social, ordre du désordre constitutif à tout ordre social.

\section{Références}

Becker, H. (1963). Outsiders. New York : Free Press.

Canada, G. (1995). Fist, knife, stick, gun. Boston : Beacon Press.

Cloward, R.A., \& Ohlin, L.E. (1960). Delinquency and opportunity. A theory of delinquent gangs. London : Routledge and Kegan Paul.

Cohen, A.K. (1955). Delinquent boys. The culture of the gang. New York: The Free Press.

Fillieule, R. (2001). Sociologie de la délinquance. Paris : PUF.

Hagedorn, J.M. (1988). People and folks : gangs, crime and the underclass in a rustbelt city. Chicago : Lakeview Press.

Hébert, J., Hamel, S., \& Savoie, G.J. (1997). Jeunesse et gangs de rue. Phase I, revue de littérature. Montréal : Service de Police de la Communauté Urbaine de Montréal, IRDS.

Huff, C.R. (1996). Gangs in America. Newbury Park : Sage.

Jankowski, M.S. (1992). Les gangs aux États-Unis. Bilan des recherches. Paris : Institut des Hautes Études de la Sécurité Intérieure.

Jankowski, M.S. (1994). Les gangs et la presse. La production d'un mythe national. Actes de la Recherche en Sciences Sociales, (101-102),110-117.

Johnstone, J.W.C. (1983). Recruitment to a youth gang. Youth and society, 14 (3), 281-300.

Juteau, D. (1999). L'ethnicité et ses frontières. Montréal : Presses de l'Université de Montréal.

Klein, M.W. (1971). Street gangs and street workers. Englewood Cliffs : Prentice Hall.

Lanctôt, N., \& Le Blanc, M. (1997). La participation à une bande marginale : continuité et conséquences. Montréal : Groupe de recherche sur les adolescents en difficulté, École de psychoéducation, Université de Montréal.

Landesco, J. (1929). Organized crime in Chicago. Part III of the Illinois Crime Survey 1929. Chicago : Illinois Association for Criminal Justice.

Lebesque, M. (1970). Comment peut-on être Breton? Essai sur la démocratie française. Paris : Seuil.

Martiniello, M. (1995). L'etbnicité dans les sciences sociales contemporaines. Paris : PUF.

Matza, D. (1964). Delinquency and drift. New York : Wiley.

Merton, R.K. (1938). Social structure and anomie. In R.K. Merton (1968), Social theory and social structure (185-214). New York : The Free Press.

Monti, D.J., \& Cummings, S. (1993). Gangs : the origins and impact of contemporary youth gangs in the United States. New York : State University of New York Press. 
Moore, J.B. (1993). Skinheads shaved for battle : a cultural history of american skinbeads. Bowling Green : Bowling Green State University Popular Press.

Moore, J.P., \& Cook, I.L. (1998). Highlights of the 1998 National Youth Gang Survey. Rockville : US Dept of Justice, Office of Juvenile Justice and Delinquency Prevention United States.

Parks, C.P. (1995). Gang behavior in the schools : reality or myth? Educational Psychology Review, 7 (1), 41-68.

Parsons, T. (1954). Essays in sociological theory. Glencoe: The Free Press.

Phillips, S.A. (1999). Wallbangin'. Graffiti and gangs in L.A. Chicago : The University of Chicago Press.

Poutignat, P., \& Streiff-Fenart, J. (1995). Théories de l'ethnicité. Paris : PUF.

Risbil, K.M., \& Davidson, W.S. (1993). Community change intervention. In A.P. Goldstein \& C.R. Huff (eds), The gang intervention bandbook (333-355). Champaign : Research Press.

Shaw, C.R., \& McKay, H.D. (1942). Juvenile delinquency and urban areas. A study of rates of delinquency in relation to differential characteristics of local communities in american cities. Chicago : The University of Chicago Press.

Skolnick, J.H. (1990). The social structure of street drug dealing. American Journal of Police, 9 (1), 1-41.

Spergel, I.A. (1992). Youth gangs : an essay review. Social Service Review, 66, $121-140$.

Spergel, I.A. (1995). The youth gang problem. New York : Oxford University Press.

Stephens, R.D. (1993). School-based interventions : safety and security. In A.P. Goldstein \& C.R. Huff (eds), The gang intervention handbook (219-255). Champaign : Research Press.

Tichit, L. (1995). Rage et défi. Approche socio-ethnographique de jeunes de banlieues populaires, à travers le mouvement hip- hop. Bordeaux : DEA Sciences de l'Éducation, Université Bordeaux II.

Tichit, L. (2001a). Quartiers Sud : racialisation et construits ethniques du racket à l'école. Ville-École-Intégration, 124 (mars), 198-206.

Tichit, L. (2001b). Le «toutes choses égales par ailleurs» en question. VilleÉcole-Intégration, (décembre), 25-34.

Trasher, F.M. (1927). The gang. A study of 1313 gangs in Chicago. Chicago : University of Chicago Press.

Walgrave, L., (1992). Délinquance systématisée des jeunes et vulnérabilité sociétale : essai de construction d'une théorie intégrative. Paris : Méridiens Klincksieck.

Weber, M. (1971). Économie et société. Paris : Plon.

Whyte, W.F. (1943). Street corner society : the social structure of an italian slum. Chicago : University of Chicago Press. 